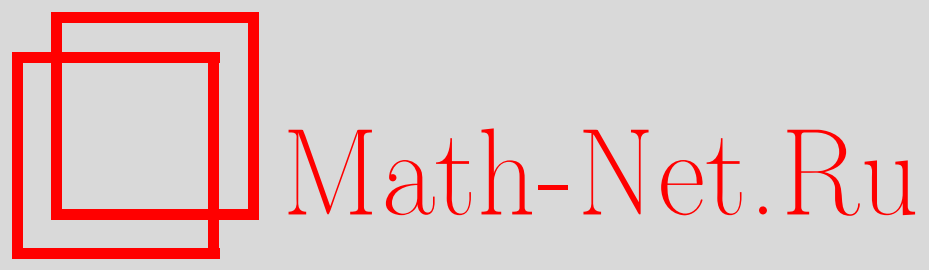

В. С. Шульман, Факторизация вполне положительных коциклов и ГНС-конструкция представлений в пространствах Понтрягина, Функи. анализ и его прил., 1997, том 31, выпуск 3, 91-94

DOI: https://doi.org/10.4213/faa486

Использование Общероссийского математического портала MathNet.Ru подразумевает, что вы прочитали и согласны с пользовательским соглашением http://www . mathnet.ru/rus/agreement

Параметры загрузки:

IP : 52.6 .47 .48

26 апреля 2023 г., 05:55:23

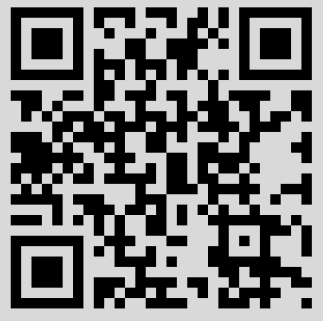


Напомним [1], что $W^{*}$-алгебра $M$ в $B(H)$ называется инъективной, если существует проектор $P$, отображающий $B(H)$ на $M$, такой, что $P(\mathbb{I})=\mathbb{I}$ и $\|P\|=1$. Вещественная алгебра $R$ называется инъективной, если обертывающая алгебра $\mathscr{U}(R)$ является инъективной (см. [5]). Пусть $\alpha$ - инволютивный *-антиавтоморфизм $W^{*}$-алгебры $M$. Под модулем отображения $\alpha$ понимается положительное вещественное число $\mu$, такое, что $\tau \cdot \alpha=\mu \cdot \tau$ (обозначение: $\mu=\bmod (\alpha))$, где $\tau$ - полуконечный след на $M$. Как известно [4], существуют два класса неизоморфных вещественных инъективных факторов типа $\mathrm{III}_{\lambda}, 0<$ $\lambda<1$, комплексно порождающих изоморфные факторы типа $\mathrm{III}_{\lambda}$. Один из них порождается инволютивным *-антиавтоморфизмом $\mathrm{c} \bmod (\alpha)=1$, а другой порождается инволютивным *-антиавтоморфизмом $с \bmod (\alpha)=\sqrt{\lambda}$. Поскольку легко построить вещественный гиперфинитный фактор $R$ типа $\mathrm{III}_{\lambda}, 0<\lambda<1$, с $\bmod (\alpha)=1$ (например, вешественный фактор Пауэрса, т. е. бесконечное тензорное произведение $2 \times 2$-вещественных матриц), из теоремы 2 вытекает

СЛЕДСТВИЕ 1. Вещественный инъективный $W^{*}$ фактор $R$ muna $\mathrm{III}_{\lambda}$, $0<\lambda<1$, является гиперфинитньм тогда и только тогда, когда $\bmod (\alpha)=1$.

Отсюда вытекает, что во втором классе содержатся инъективные, но не гиперфинитные вещественные факторы типа $\mathrm{III}_{\lambda}, 0<\lambda<1$, т. е. имеет место

СлЕдСТВИЕ 2. Для вещественных факторов понятия инъективности $u$ гиперфинитности неэквивалентны.

Напомним [1], что в комплексном случае эти понятия совпадают.

\title{
ЛИТЕРАТУРА
}

1. Connes A. Ann. Math., 104, No. 1, 73-115 (1976). 2. Stacey P. London Math. Soc. (3), 47, 275-284 (1983). 3. Рахимов А. А., Усманов ШШ. М. Функц. анализ и его прил., 29, вып. 1, 87-89 (1995). 4. Giordano T. J. Funct. Anal., 51. No. 3, 326-360 (1983). 5. Аюпов III. A. Классификация и представление упорядоченных йордановых алгебр. Изд-во «ФАН», Ташкент, 1986. 6. Stormer E. Duke Math. J., 47, No. 1. 145-153 (1980). 7. Elliott G. A., Woods E. J. Proc. Am. Math. Soc., 60, 175-179 (1976).

Университет Мировой Экономики и Дипломатии,

Республика Узбекистан, г. Ташкент

Поступило в редакцию e-mail: root@im.tashkent.su

16 апреля 1996 г.

УДК 517

\section{Факторизация вполне положительных коциклов и ГНС-конструкция представлений в пространствах Понтрягина}

\author{
(C) 1997. В. С. ШульмАн
}

Пространство Понтрягина $\Pi_{k}$ - это линейное пространство $H$ с индефинитным скалярным произведением $[\cdot, \cdot]$, допускающее разложение в сумму двух $J$-ортогональных (символ $J$ перед термином означает, что в его определении 
$[\cdot, \cdot]$ играет ту же роль, какую обычно играет скалярное произведение) подпространств, одно из которых $k$-мерно и отрицательно $([x, x]<0$ при $x \neq 0)$, а другое положительно и полно относительно нормы $[x, x]^{1 / 2}$. В алгебре $\mathscr{B}(H)$ всех непрерывных линейных операторов в $H$ вводится инволюция $A \rightarrow A^{\#}$ (переход к $J$-сопряженному оператору). В ряде задач (классификация элементарных представлений полупростых групп, ортогонализация представлений инволютивных алгебр, построение моделей алгебраической теории поля) возникают вопросы классификации $J$-унитарных представлений групп и $J$-симметричных представлений *-алгебр в $\Pi_{k}$. Изучению этих вопросов посвящены, в частности, работы [1-7]. Известно $[6,7]$, что $J$-симметричные представления $C^{*}$-алгебр в $\Pi_{k}$ и групповых алгебр в $\Pi_{1}$ подобны *-представлениям в гильбертовом пространстве. В [5] показано, что столь же просто описываются невырожденные (не имеющие нейтральных инвариантных подпространств) представления почти эрмитовых *-алгебр, т.е. алгебр, у которых самосопряженные элементы с вещественным спектром плотны в пространстве всех самосопряженных элементов. В настоящей работе изучается класс представлений, в естественном смысле противоположный классу невырожденных представлений. Будем называть $J$-симметричное представление в $\Pi_{k}$ max-представлением, если оно обладает инвариантным нейтральным подпространством размерности $k$ (т. е. максимально возможной размерности). Мы построим аналог ГНС-конструкции и докажем его универсальность в классе max-представлений почти эрмитовых алгебр. В заключение будут рассмотрены вопросы разложимости представлений в зацепленные суммы относительно просто устроенных (расщепимых) слагаемых.

Начнем с общего результата о факторизуемости вполне положительных 2-коциклов, который можно рассматривать как когомологически оснащенную версию теоремы Стайнспринга [9].

Пусть $\lambda$ - представление банаховой *-алгебры $\mathscr{A}$ в гильбертовом пространстве $\mathscr{H}$, а $\lambda^{\sim}$ - сопряженное представление $\left(\lambda^{\sim}(a)=\left(\lambda\left(a^{*}\right)^{*}\right)\right)$; тогда $\mathscr{B}(\mathscr{H})$ является $\mathscr{A}$-бимодулем относительно действия

$$
a X b=\lambda^{\sim}(a) X \lambda(b) \quad(a, b \in \mathscr{A}, X \in \mathscr{B}(\mathscr{H}))
$$

и $\mathscr{B}(\mathscr{H})$-когомологии можно реализовать в пространствах непрерывных полилинейных отображений из $\mathscr{A}$ в $\mathscr{B}(\mathscr{H})$. Аналогично определяются когомологии с коэффициентами в $\mathscr{B}\left(\mathscr{H}_{1}, \mathscr{H}_{2}\right)$ для любых $\mathscr{A}$-модулей $\mathscr{H}_{1}, \mathscr{H}_{2}$. Напомним, что билинейное отображение $\Phi: \mathscr{A} \times \mathscr{A} \rightarrow \mathscr{B}(\mathscr{H})$ называется вполне положительным, если $\sum_{i, j}\left(\Phi\left(a_{i}^{*}, a_{j}\right) \xi_{i}, \xi_{j}\right) \geqslant 0$ для любых $a_{i} \in \mathscr{A}, \xi_{i} \in \mathscr{H}$.

Теорема 1. Пусть $\Phi: \mathscr{A} \times \mathscr{A} \rightarrow \mathscr{H}$ - вполне положительньй 2-коиикл; тогда суиествуют и ( с точностью до естественно понимаемой эквивалентности) единственнь гильбертово пространство $\mathfrak{H}_{\Phi}$, представление $\pi_{\Phi}$ алгебрьл $\mathscr{A}$ в $\mathfrak{H}_{\Phi}$ u 1-коиикл $p_{\Phi}: \mathscr{A} \rightarrow \mathscr{B}\left(\mathscr{H}, \mathfrak{H}_{\Phi}\right)$, maкие, что подпространство $p_{\Phi}(\mathscr{A}) \mathscr{H}$ плотно в $\mathfrak{H}_{\Phi}$ и

$$
\Phi(a, b)=p\left(a^{*}\right) p(b)
$$

для любых $a, b \in \mathscr{A}$.

ОПрЕДЕЛЕниЕ. Линейное непрерывное отображение $\gamma: \mathscr{A} \rightarrow \mathscr{B}(\mathscr{H})$ называется $\lambda$-диссипацией, если его кограница $d \gamma$ - вполне положительное отображение. 
Заметим, что 0-диссипации - это вполне положительные отображения в смысле Стайнспринга [9].

Нетрудно показать, что всякая $\lambda$-диссипация становится эрмитовой после прибавления некоторого 1-коцикла; мы будем рассматривать только эрмитовы $\lambda$ диссипации.

Пусть $\gamma$ - некоторая $\lambda$-диссипация. Тогда $\Phi=d \gamma$ - вполне положительный 2 -коцикл и в силу теоремы 1 существуют пространство $\mathfrak{H}=\mathfrak{H}_{\Phi}$, представление $\pi=\pi_{\Phi}$ и 1-коцикл $p=p_{\Phi}$, такие, что выполнено (1). В правой сумме $H=$ $\mathscr{H}+\mathfrak{H}+\mathscr{H}$ определим представление $n_{\lambda, \gamma}$ алгебры $\mathscr{A}$ формулой

$$
n_{\lambda, \gamma}(a)(\xi+x+\eta)=\left(\lambda^{\sim}(a) \xi+p(a) x+\gamma(a) \eta\right)+(\pi(a) x+p(a) \eta)+\lambda(a) \eta .
$$

Нетрудно проверить, что относительно индефинитного скалярного произведения $\left[\xi_{1}+x_{1}+\eta_{1}, \xi_{2}+x_{2}+\eta_{2}\right]=\left(\xi_{1}, \eta_{2}\right) \mathscr{H}+\left(x_{1}, x_{2}\right)_{\mathfrak{H}}+\left(\eta_{1}, \xi_{2}\right) \mathscr{H}$ пространство $H$ является пространством $\Pi_{k}$ с $k=\operatorname{dim} \mathscr{H}$ (пространством Крейна, если $\operatorname{dim} \mathscr{H}=\infty)$ и представление $n_{\lambda, \gamma} J$-симметрично.

Из теоремы 1 нетрудно вывести (трактуя когомологически компоненты в стандартной операторно-матричной реализации представления), что всякое maxпредставление банаховой *-алгебры в $\Pi_{k} J$-унитарно эквивалентно некоторому $n_{\lambda, \gamma}$. Это позволяет описать все $\Pi_{k}$-представления довольно широкого класса алгебр.

СЛЕДСТВИЕ 2. Если $A$ - почти эрмитова банахова *-алгебра, не имеющая нетривиальных конечномерных представлений, то всякое, с точностью до Ј-унитарной эквивалентности и до тривиального прямого слагаемого, ее $\Pi_{k}$-представление задается вполне положительньм отображением $\gamma: A \rightarrow$ $M_{k}(\mathbb{C})$ по формуле $T=n_{0, \gamma}$.

В частности, представления почти эрмитовых алгебр в $\Pi_{1}$ строятся по положительным функционалам.

Обратимся к вопросу о разложимости $\Pi_{k}$-представления в сумму простейших.

ТеоремА 3. Пусть $\pi$ - представление почти эрмитовой *-алгебры $\mathscr{A}$ в $\Pi_{k}$-пространстве $H, L$ - его максимальное нейтральное инвариантное подпространство и $\lambda$-ограничение $\pi$ на L. Если образ представления $\lambda$ — полупростая алгебра, а ядро имеет ограниченную аппрокисмативную единичу, то $H=L+M+\mathfrak{H}$, где все слагаемье инвариантнь, $M$ нейтрально и ограничение $\pi$ на $\mathfrak{H}$ подобно *-представлению.

Отказ от условия полупростоты алгебры $\lambda(\mathscr{A})$ создает лишь «конечномерные препятствия» - ограничивая $\pi$ на подалгебру конечной коразмерности $\operatorname{Ker} \lambda \cap \operatorname{Ker} \lambda^{\sim}$, можно считать, что $\lambda=0$. Существеннее второе ограничение, не выполняющееся в таких важных примерах, как представления дифференциальных [8] и (неаменабельных) групповых алгебр. Условимся называть представление расщепимыл, если оно лишь конечномерным прямым слагаемым отличается от представления, подобного *-представлению.

ТЕоремА 4. Для того чтобы представление $n_{\lambda, \gamma}$ было расщепимым, необходимо и достаточно, чтобь отображение $d \gamma$ было непрерьвныл относительно обертьввающей $C^{*}-$ нормь $\|\cdot\|_{0}$ алгебры $\mathscr{A}$.

Рассмотрим теперь вопрос о разложимости ГНС-представления в зацепленную сумму расщепимых представлений. 
ОПРеДЕЛЕНИЕ. Разложением П ${ }_{k}$-пространства $H$ в сумму, зачепленную по нейтральному подпространству $L$, называется такой набор подпространств $H_{i}$, содержащих $L$, что подпространство $\mathscr{E}=\bigcap_{i} H_{i}$ невырожденно, а подпространства $L_{i}=H_{i} \cap L^{\perp}$ взаимно $J$-ортогональны и порождают $L^{\perp}$.

Из определения легко следует, что $\operatorname{dim} \mathscr{E}=2 \operatorname{dim} L$ и все подпространства $H_{i}$ невырожденны. Обозначим через $P_{i} J$-ортогональные проекторы на $H_{i}$ в $H$. Если $\pi$ есть $J$-симметричное представление алгебры $\mathscr{A}$ в $H$, относительно которого все $L_{i}$ инвариантны, то, полагая $\pi_{i}(a)=P_{i} \pi(a) \mid H_{i}$, мы зададим $J$-симметричные представления $\pi_{i}$ в $H_{i}$. В этом случае говорят, что $\pi$ разложено в зацепленную по $L$ сумму представлений $\pi_{i}$.

Теорема 5. Для того чтобы представление $n_{\lambda, \gamma}$ допускало разложение в зачепленную сумму расщепимых представлений, необходимо и достаточно, чтобь вполне положительное отображение $d \gamma$ было полунепрерывнылм снизу относительно нормы $\|\cdot\|_{0}$ (т.е. чтобы из того, что $\left\|a_{n}-a\right\|_{0} \rightarrow 0$, следовало $\left.\left\|d \gamma\left(a^{*}, a\right)\right\| \leqslant \lim \sup \left\|d \gamma\left(a_{n}^{*}, a_{n}\right)\right\|\right)$.

Автор признателен А. И. Логинову и Э. В. Киссину за полезные обсуждения рассмотренных в работе вопросов, а также рецензенту за ценные замечания.

\section{ЛитеРАТУРА}

1. Наймарк М. А. Изв. АН СССР, сер. матем., 30, №5, 1111-1132 (1966). 2. Исмиагилов Р. С. Изв. АН СССР, сер. матем., 30, №3, 497-522 (1966). 3. Исмагилов Р. С. Матем. заметки, 35, №1, 99-105 (1984). 4. Наймарк М. А., Исмагилов Р. С. Представления групп и алгебр в пространствах с индефинитной метрикой. Итоги науки и техники. Математический анализ, ВИНИТИ, 1968, с. 73-105. 5. Kissin E., Loginov A. I., Shulman V. S. Derivations of $C^{*}$-algebras and almost hermitian representations on $\Pi_{k}$-spaces. Pacif. J. Math. (в печати). 6. Шульман В. С. Матем. заметки, 22, №4, 583-592 (1977). 7. Шульман В. С. Функц. анализ и его прил., 14, вып. 2, 93-94 (1980). 8. Kissin E., Shulman V. S. Proc. Edinburgh Math. Soc., 37, 399-422 (1984). 9. Stinespring W. F. Proc. Am. Math. Soc., 6, 211-216 (1965).

Вологодский государственный естественно-математический лицей

Поступило в редакцию 12 октября 1995 г. 